\title{
LEVEL SETS LEMMAS AND UNICITY OF CRITICAL POINT OF INVARIANTS, TOOLS FOR LOCAL STABILITY AND TOPOLOGICAL PROPERTIES OF DYNAMICAL SYSTEMS
}

\author{
GUY BASTIEN AND MARC ROGALSKI
}

Dedicated to Professor Mustafa Kulenović on the occasion of his 60th birthday

\begin{abstract}
We prove that the level curves of some differentiable functions of two variables with unique critical point are diffeomorphic to the circle $\mathbb{T}$, and show how this result can be used in the study of local stability of dynamical systems in dimension 2 with invariant function, without using the Hessian. We extend the results to the level sets of an invariant function of dynamical systems, with a synthesis exposition of examples of improvements of previously studied order $q$ difference equations with invariant. In fine we present some differential tools for the study of the topological nature of invariant level sets in dimension at least three.
\end{abstract}

\section{INTRODUCTION}

We will highlight the role of level sets and unicity of critical point of invariant functions in place of the use of Hessian, in the study of local stability of a large class of dynamical systems, in particular those associated to difference equations.

First we study the case of dimension 2, where special arguments permit to identify the topological nature of the level curves. Then we study the general case of dynamical systems with invariant in connected locally compact topological spaces. We also show how our result can be applied to some order $q$ difference equations previously studied by several authors. In the last section, we present some tools of differential geometry which permit us to study the topological nature of level sets of invariants of order $q$ difference equations.

2000 Mathematics Subject Classification. 37E75, 39A20, 39A30.

Key words and phrases. Difference equations, dynamical systems, local stability, level sets. 


\section{The Level CuRVes Lemma}

The following result can be used in the study of some planar dynamical systems.

Lemma 1 (The level curves lemma). Let $\Omega \neq \emptyset$ be a connected and simply connected open subset of $\mathbb{R}^{2}$, and $V: \Omega \rightarrow \mathbb{R}$ a $C^{1}$ function satisfying the two conditions :

(1) The limit $V(\infty) \leq+\infty$ of $V(x)$ when $x$ tends to the infinity point of $\Omega$ exists, and $V<V(\infty)$ in $\Omega$;

(2) $V$ has exactly one critical point $\mathbf{p}$ in $\Omega$.

Then we have the following properties:

(a) $V$ attains its strict minimum in $\Omega$ at the point $\mathbf{p}$;

(b) For every $\lambda \in] V(\mathbf{p}), V(\infty)[$, the level set $C(\lambda):=\{x \mid V(x)=\lambda\}$ is not empty and is a $C^{1}$ 1-dimensional compact manifold;

(c) This curve $C(\lambda)$ is a $C^{1}$ Jordan curve which is diffeomorphic to the circle (and thus connected); its interior contains the point $\mathbf{p}$, is exactly the connected set $\{x \mid V(x)<\lambda\}$, and is homeomorphic to the open unit disk $\mathbb{D}$; its exterior is exactly the set $\{x \mid V(x)>\lambda\}$, and is homeomorphic to $\mathbb{C} \backslash \mathbb{D}$; moreover $C(\lambda)$ is the boundary of these two sets.

Proof. We will use three well-known but not easy facts.

Fact 1. The Jordan-Schönflies' theorem: Every homeomorphism of the circle $\mathbb{T}$ into the plane has an extension in a homeomorphism of the plane onto itself. This contains the classical Jordan's theorem on a Jordan curve $\Gamma$ : interior homeomorphic to $\mathbb{D}$, exterior homemorphic to $\mathbb{C} \backslash \overline{\mathbb{D}}, \Gamma$ is the boundary of these two sets (see [8]).

Fact 2. The characterisation of $\mathbb{T}$ as a manifold: Every $C^{1}$ 1-dimensional compact and connected manifold (here in the plane) is diffeomorphic to the circle (see [15]).

Fact 3. A particular case of Riemann's theorem: Every connected and simply connected open set in the plane is homeomorphic to the plane (see $[16])$.

(a) By (1), $V$ has a minimum in $\Omega$, which is strict by (2), and there is no local extremum to $V$ except the point $\mathbf{p}$.

(b) If $\lambda \in] V(\mathbf{p}), V(\infty)[, C(\lambda)$ is not empty by the connexity of $\Omega$, compact by (1); it is a $C^{1} 1$-dimensional manifold by (2) and the implicit function theorem (because $\mathbf{p} \notin C(\lambda)$ ). 
(c) $(*)$ Since a manifold is locally connected, the connected components of $C(\lambda)$ are open in $C(\lambda)$ (and of course compact), and by compactness $C(\lambda)$ has only finite number of connected components. Each of these components is diffeomorphic to the circle, by Fact 2 .

(**) By Facts 1 and 3, each of these components is the boundary of its connected interior, and the union of these two sets is homeomorphic to $\overline{\mathbb{D}}$, and then compact in $\Omega$. By $(*)$ there is at least one of these components, say $\Gamma$, whose interior $I$ contains no other component, and so $I \subset\{V \neq \lambda\}$. By the connexity of $I$, we have in $I: V>\lambda$ or $V<\lambda$. The first case is excluded, by compactness and because $V$ has no local maximum by the proof of (a). So $V$ has a local minimum in $I$ which is necessarily $\mathbf{p}$ by (2).

$(* * *)$ We will prove that $\Gamma=C(\lambda)$. If not, there exist other connected components of $C(\lambda)$. There are two possibilities. First, one of these components, say $\Gamma^{\prime}$ (diffeomorphic to the circle), has its interior $I^{\prime}$ which contains no other component, is connected, with $\overline{I^{\prime}}=I^{\prime} \cup \Gamma^{\prime}$ compact, by Facts 1 and 3, and with $\bar{I}^{\prime} \subset E$, the exterior of $\Gamma$ (Fact 1 and choice of $\Gamma$ ). By the same reasoning than in $(* *), I^{\prime} \subset\{V<\lambda\}$. So $V$ would have a critical point in $E$, and this is impossible. In the other possibility, all the other components surround $\Gamma$, and there is one, say $\Gamma^{\prime}$, which surrounds $\Gamma$, and no other component by the impossibility of the first case. So in the non-empty "annulus" between $\Gamma^{\prime}$ and $\Gamma$ (use Fact 1 ) the function $V$ must have a local extremum and so a critical point different from $\mathbf{p}$, and this is impossible. In fine, one has $\Gamma=C(\lambda)$.

Now, Fact 1 implies the end of the point (c) of the lemma.

Remark. The proof does not work if the hypothesis " $\Omega$ is connected and simply connected" is omitted: in this case, some steps of the proof do not work; for example, the arguments of compactness in points $(* *)$ and $(* * *)$ of step (c) of the proof fail. But in fact one can prove that the hypothesis (1) and (2) on the function $V$ imply that $\Omega$ is connected and simply connected (see the Fact 4 below). In practice, this result is often unuseful, because the open sets which tipically appear in the use of the lemma for studying dynamical systems are naturally connected and simply connected. Nevertheless we give a sketch of proof of this property.

Fact 4. Let be $\Omega \neq \emptyset$ an open subset of $\mathbb{C}$. Then the following assertions are equivalent:

(1) $\Omega$ is connected and simply connected ;

(2) There exist a $C^{1}$ function $V: \Omega \mapsto \mathbb{R}$ satisfying

(a) $\lim _{z \rightarrow \infty \text { in } \Omega} V(z):=V(\infty) \leq+\infty$ exists, and $V<V(\infty)$ in $\Omega$;

(b) $V$ has only one critical point in $\Omega$. 
Hint for the proof. The implication (1) $\Longrightarrow(2)$ is easy: it is obvious if $\Omega=\mathbb{C}$ (take $V(x, y):=x^{2}+y^{2}$ ), and if not take $V(x, y):=|\phi(x+i y)|^{2}$ where $\phi$ is the conformal map of $\Omega$ onto $\mathbb{D}$ (and use Cauchy-Riemann equations).

For the converse, it is first easy to see that $\Omega$ is connected: if not, $V$ would have a critical point in every connected component and so at least two critical points, contrary to the hypothesis.

For the second part of the converse, we will see that if $\Omega$ is connected but not simply connected, then every $C^{1}$ function $V: \Omega \mapsto \mathbb{R}$ satisfying (a) has at least 2 critical points.

We know that $\partial \Omega\left(\right.$ in $\left.\mathbb{C}^{\infty} \approx \mathbb{S}^{2}\right)$ is not connected (see [16], Theorem 13.11), and so if $\lambda$ is near $V(\infty)$ the level set $\{V=\lambda\}$ is not connected (if $\partial \Omega$ splits into two disjoint compact sets $H_{1}$ and $H_{2}$, and if we choose two open sets $U_{1}$ and $U_{2}$ such that $H_{1} \subset U_{1}, H_{2} \subset U_{2}$ and $\bar{U}_{1} \cap \bar{U}_{2}=\emptyset$, then $\{V=\lambda\}$ cuts $\bar{U}_{1}$ and $\left.\bar{U}_{2}\right)$. But if $m:=\min V=V(p)$, then $p$ is a critical point, and one can suppose that it is isolated. One can see, by the same method as in point (c) of the proof of Lemma 1, that if $\lambda$ is near $m$, then the level set $\{V=\lambda\}$ is homeomorphic to the circle, and so is connected. Now, if there were no other critical point than $p$, a classical result of differential geometry (see Section IV, Fact 6, and [15], Ch.3, Theorem 50) asserts that all the level sets $\{V=\mu\}$ would be homeomorphic, and this is a contradiction.

\section{Applications of the level Curves lemma to Dimension 2 DYNAMICAL SYSTEMS}

First we give a general result on dynamical systems in dimension 2. Then we give examples of previously studied difference equations were the general result can be used.

\subsection{The general result in dimension 2 .}

Notations. We look at an order 2 difference equation

$$
u_{n+2}=\phi\left(u_{n+1}, u_{n}\right) \text {, }
$$

where $\phi$ is such that the map $F:(x, y) \mapsto(\phi(x, y), x)$ acts continuously on an open set $\Omega \subset \mathbb{R}^{2}$. More generally, we look at a system of two order 1 difference equations

$$
x_{n+1}=f\left(x_{n}, y_{n}\right), \quad y_{n+1}=g\left(x_{n}, y_{n}\right),
$$

where the map $F=(f, g)$ acts continuously on an open set $\Omega \subset \mathbb{R}^{2}$.

If $M_{n}=\left(u_{n+1}, u_{n}\right)$, or $M_{n}=\left(x_{n}, y_{n}\right)$, then $F\left(M_{n}\right)=M_{n+1}$, and $(\Omega, F)$ is the dynamical system associated to equation (1) or to system (2).

Proposition. Consider equation (1) or system (2). 
We suppose that there is a $C^{1}$ function $G: \Omega \rightarrow \mathbb{R}$ which is invariant, that is $G \circ F=G$ in $\Omega$, and that $\Omega, G$ and $F$ satisfy three hypothesis:

(i) $\lim _{M \rightarrow \infty \text { in } \Omega} G(M):=G(\infty) \leq+\infty$ exists, and $G<G(\infty)$ in $\Omega$;

(ii) $G$ has at most one critical point $L$ in $\Omega$ (so its minimum is $G(L):=$ $\left.G_{m}\right)$;

(iii) F has at most one fixed point.

Then the following properties happen:

(a) The point $L$ is the (unique) fixed point of $F$;

(b) For $K \in] G_{m}, G(\infty)$ the level set $\mathcal{C}_{K}:=\{G=K\}$ is non-empty and is a $C^{1}$ Jordan curve which is diffeomorphic to the circle $\mathbb{T}$, and surrounds the equilibrium $L$;

(c) The orbit $\left(M_{n}=F^{n}\left(M_{0}\right)\right)_{n}$ is included in $\mathcal{C}_{G\left(M_{0}\right)}$, and if $M_{0} \neq L$ then the sequence $M_{n}$ does not converge;

(d) The equilibrium $L$ is locally stable;

(e) If $\Omega \subset \mathbb{R}_{*}^{+2}$, then the solutions of equation (1) or of system (2) are permanent.

Hint for the proof. First, by Fact $4, \Omega$ is connected and simply connected. Then $G(F(L))=G(L)=G_{m}$, and so $G$ is minimum at $F(L)$, so $F(L)$ is a critical point, and thus is the point $L$. Point (b) follows from the level curves lemma. Point (c) follows from the invariant nature of $G$ and thus of the $\mathcal{C}_{K}$. From the level curves lemma, for $\left.K \in\right] G_{m}, G(\infty)[$, the sets $\{G<K\}$ are open, their closures are the compact sets $\{G \leq K\}$, and the intersection of these sets reduces to $\{L\}$; so the sets $\{G<K\}$ form a fundamental system of neighborhoods of $L$ which are stable under the action of $F$. So $L$ is locally stable. In fine if $\Omega \subset \mathbb{R}_{*}^{+2}$, by the compactness of the $\mathcal{C}_{K}, m:=\min _{(x, y) \in \mathcal{C}_{K}} y>0$ and $M:=\max _{(x, y) \in \mathcal{C}_{K}} y<+\infty$, and so the solutions of (1) or (2) are permanent. Of course, if $\Omega$ is not included in $\mathbb{R}_{*}^{+2}$, the conclusion is only that each solution is bounded.

Notice that the local stability does not use the Hessian. In some papers the hypothesis of the previous proposition are proved, but unnecessary tedious calculations for study the Hessian are made.

If equations (1) or (2) satisfy the proposition, some questions are:

Problems. Is the restriction of the map $F$ to the level curve $\mathcal{C}_{K}$ conjugated to a rotation on the circle $\mathbb{T}$ ? If yes, what about the angle $\theta(K)$ of the rotation? About periodic orbits or dense orbits in $\mathcal{C}_{K}$ ? What are the possible periods of solutions? Is there a sort of chaotic behavior of the system?

2.2. Example: Lyness' difference equation and some generalizations. Here we give some examples of discrete dynamical systems for which 
our result may facilitate the study of the topology of the level sets of the first integral associated to them.

The classical order 2 Lyness' difference equation, which is $u_{n+2} u_{n}=$ $a+u_{n+1}, a>0, u_{0}, u_{1}>0$. In this case, $\Omega=\mathbb{R}_{*}^{+2}, F(x, y)=((a+x) / y, x)$, the invariant function is $G(x, y)=(x+1)(y+1)(a+x+y) / x y$, and each level set is the positive component of some elliptic cubic curve. From the level curves lemma, such level set is diffeomorphic to the unit circle. The behavior of the associated dynamical system was in part conjectured in [1] and proved first in [17], a non published paper, and discovered again later, but independently, in [2] and in [11]. In paper [2] there is some new accent on a form of chaotic behavior of the system. A deep study of rational periodic solutions (when $a$ is rational) is given recently in [12].

(a) Many examples which generalize Lyness' case are the equations in $\mathbb{R}_{*}^{+}$

$$
u_{n+2} u_{n}=\frac{a+b u_{n+1}+c u_{n+1}^{2}}{c+d u_{n+1}+e u_{n+1}^{2}}, u_{1}, u_{0}>0 .
$$

The invariant is

$$
G(x, y)=\left[e x^{2} y^{2}+d x y(x+y)+c\left(x^{2}+y^{2}\right)+b(x+y)+a\right] / x y,
$$

and the level curves are the quartic curves

$$
e x^{2} y^{2}+d x y(x+y)+c\left(x^{2}+y^{2}\right)+b(x+y)+a-K x y=0 .
$$

These equations were introduced in [3], and the complete study is made in [3] and [4]. The level sets are diffeomorphic to circles by the level curves lemma; they are conics, or positive component of cubic curves, or positive component of quartic curves. In the two last cases, the level curves lemma is more simple than the use of Weierstrass' function $\wp$.

(b) An interesting example where one can use the proposition is given in [9], which studies the 2-periodic order 2 Lyness' equation

$$
u_{n+2} u_{n}=a_{n}+u_{n+1} \text {, with } a_{2 k+1}=a \text { and } a_{2 k}=b .
$$

The associated map $F$ is

$$
\begin{aligned}
F:\left(u_{2 k+1}, u_{2 k+2}\right) \mapsto\left(u_{2 k+3}, u_{2 k+4}\right): & (x, y) \\
& \mapsto F(x, y)=\left(\frac{a+y}{x}, \frac{a+b x+y}{x y}\right),
\end{aligned}
$$

and the invariant is

$$
G(x, y)=\left[a x^{2} y+b x y^{2}+b x^{2}+a y^{2}+\left(b^{2}+a\right) x+\left(b+a^{2}\right) y+a b\right] / x y .
$$


(c) Another example is given in [5], where one studies the equation $u_{n+2}+$ $u_{n}=\frac{\alpha+\beta u_{n+1}}{1+u_{n+1}^{2}}($ with conditions on $\alpha, \beta)$; the invariant is $G(x, y)=x^{2} y^{2}+$ $x^{2}+y^{2}-\beta x y-\alpha(x+y)$.

In all examples of this part, answers to the problems evoked in Section I are given, see the corresponding references.

\section{An ABSTRACT LEVEL SETS LEMma, SOME EXAMPLES OF APPLICATIONS}

In this section, we give a more or less classic general result for a dynamical system with invariant. Then we give examples of applications to order $q$ difference equations.

\subsection{The general assertion.}

Lemma 2 (The level sets lemma). Let be $X$ a connected and locally compact space. Let be $F: X \longrightarrow X$ and $G: X \longrightarrow \mathbb{R}$ two continuous maps. We suppose that the following conditions hold:

(i) $G$ has a strict minimum $G_{m}$ at a point $L$, and no other local minimum;

(ii) $\forall x \in X, G \circ F(x)=G(x)$ ( $G$ is an invariant function);

(iii) $F$ has at most one fixed point.

(iv) $G(\infty):=\lim _{x \rightarrow \infty \text { in } X} G(x) \leq+\infty$ exists, and $G(x)<G(\infty)$ in $X$.

Then we have:

(1) the point $L$ is the (unique) fixed point of $F$;

(2) for $K \in] G_{m}, G(\infty)\left[\right.$, the level set $\mathcal{C}_{K}:=\{G=K\}$ is compact and non-empty;

(3) if $M_{0} \in X$ let be $M_{n+1}=F\left(M_{n}\right)$ the points of the orbit of $M_{0}$ under $F$; then $M_{n} \in \mathcal{C}_{G\left(M_{0}\right)}$, and if $M_{0} \neq L$, then the sequence $\left(M_{n}\right)$ does not converge.

(4) the equilibrium point $L$ is locally stable.

(5) for $K \in] G_{m}, G(\infty)\left[\right.$, the set $\mathcal{C}_{K}$ is the boundary of the open set $U_{K}:=$ $\{G<K\}$ which is a connected relatively compact set.

The proof of this lemma is given in [4]. It gives a tool for proving local stability only with properties of the level sets of the invariant, in the spirit of the use of Lyapunov functions (see [14]): the key is the existence of fundamental neighborhoods of $L$ which are invariant.

3.2. Applications to dynamical systems in $\mathbb{R}^{q}$, linked to difference equations. In the applications, hypothesis (i) of Lemma 2 follows often from the following property:

(UCP) The invariant function $G$ is of class $C^{1}$ and has a unique critical point in $X$. 
(a) The order $q$ Lyness' difference equation

$$
u_{n+q}=\left[a+u_{n+q-1}+\cdots+u_{n+1}\right] / u_{n},
$$

with $a>0, u_{0}, u_{1}, \ldots, u_{q-1}>0$, is the first example. In this case, the space $X$ is $\mathbb{R}_{*}^{+q}$, and $F$ is defined by

$$
F\left(x_{1}, x_{2}, \ldots, x_{q}\right)=\left(\left[a+x_{1}+\cdots+x_{q-1}\right] / x_{q}, x_{1}, x_{2}, \ldots, x_{q-1}\right),
$$

if we take $M_{n}=\left(u_{n+q-1}, \ldots, u_{n}\right)$. It is classical that there is a first invariant function

$G\left(x_{1}, \ldots, x_{q}\right)=\left[\left(x_{1}+1\right)\left(x_{2}+1\right) \ldots\left(x_{q}+1\right)\left(a+x_{1}+x_{2}+\cdots+x_{q}\right)\right] / x_{1} x_{2} \ldots x_{q}$.

It is proved in [6] that $G$ has property (UCP) and that the dynamical system $\left(\mathbb{R}_{*}^{+q}, F\right)$ satisfies the hypothesis of Lemma 2 . So the equilibrium is locally stable, and the solutions are permanent. In the case $q=3$ a study is given for the first time in [10], and an other point of view is in [6]; in this two papers, answers to the problems of part 2.1 are given.

(b) The $q$-periodic order $q$ Lyness equation

$$
u_{n+q}=\left(1+u_{n+q-1}+\cdots+u_{n+1}\right) / p_{n} u_{n}
$$

with $p_{n}>0$ and $q$-periodic, and with $u_{0}, u_{1}, \ldots, u_{q-1}>0$, is the second example. If $q=2$ (and only in this case) it is the same problem as in (b) of Section II.2. This equation is studied in [13]. The authors prove that if one put $x_{n}^{(0)}=u_{q n}, x_{n}^{(1)}=u_{q n-1}, \ldots, x_{n}^{(q-1)}=u_{q n-q+1}$, we obtain a dynamical system in $\mathbb{R}_{*}^{+q}$ which has an invariant function

$G\left(x_{0}, \ldots, x_{q-1}\right)=\left(1+\frac{1}{r_{n-q+1} x_{n-q+1}}\right) \ldots\left(1+\frac{1}{r_{n} x_{n}}\right)\left(1+x_{n}+\cdots+x_{n-q+1}\right)$

where the $r_{i}$ are the $q$ values of the periodic function $n \mapsto p_{n}$. There is a unique equilibrium and (UCP) is satisfied. Moreover, property (iv) of the Lemma 2 is easy to prove. So the local stability is true, and the tedious calculations in [13] with the Hessian are unnecessary.

\section{Differential and GeOmetric tools for LeVel Sets of INVARIANTS IN $\mathbb{R}^{q}$ WHEN $q \geq 3$}

Lemma 2 is a good tool for proving local stability, but it says nothing about the topological nature of the level sets of invariant functions when $q \geq 3$. A natural question which generalizes the 2 -dimensional situation is the following:

Question. Under hypothesis of Lemma 2 for $X$ an open subset of $\mathbb{R}^{q}$, are the level sets of the invariant homeomorphic to the sphere $\mathbb{S}^{q-1}$ ? 
If (UCP) is true, the level sets $\{G=K\}$ are manifolds. In this case, the following result of Reeb may be useful (see [15] ch.III H).

Fact 5 (Reeb's theorem). If $\mathcal{C}$ is a $C^{1} k$-dimensional compact manifold, and if there is a $C^{1}$ numerical function $f$ on $\mathcal{C}$ which has exactly 2 critical points (which can even be degenerated), then $\mathcal{C}$ is homeomorphic to the sphere $\mathbb{S}^{k}$.

For example, the first invariant $G$ of order $q$ Lyness' difference equation has easily (UCP) property (see [6]), and so for $K \in] G_{m}, G(\infty)$ [ the level sets $\mathcal{C}_{K}:=\{G=K\}$ are differentiable manifolds. It is proved easily in [6] that the function $f\left(x_{1}, \ldots, x_{q}\right):=\sum_{i=1}^{q} x_{i}$ has exactly two critical points on $\mathcal{C}_{K}$. So $\mathcal{C}_{K}$ is homeomorphic to the $(q-1)$-dimensional sphere.

In some cases it is possible to prove the homeomorphism to spheres of the level sets $\{H=K\}$ of an invariant $H$ (satisfying Lemma 2) only when $K$ is sufficiently near to the minimum $H_{m}$ of $H$ (at the fixed point $L$ ). In this case, property (UCP) permits to have an homeomorphism to spheres for all values of $K \in] H_{m}, H(\infty)$ [, from the following useful result (see [15] Ch. 3 Theorem 50):

Fact 6. Let be $V$ a differential manifold, and $f: V \mapsto \mathbb{R}$ a smooth function, such that

(i) $f$ has a unique critical point $\alpha$, at its absolute minimum $m=f(\alpha)$;

(ii) For every $\lambda$, $\mu$ such that $m \leq \lambda<\mu$, the subset $V_{\lambda}^{\mu}:=\{x \mid \lambda \leq$ $f(x) \leq \mu\}$ is compact.

Then there is a diffeomorphism of $V$ which maps $V_{m}^{\lambda}$ onto $V_{m}^{\mu}$.

For example, in [6] and [7] this method is used for proving that for $q=3,4$ and 5 the level sets of the second invariant $H$ for order $q$ Lyness' difference equation (6) are homeomorphic to spheres, where the second invariant is given by

$H\left(x_{1}, \ldots, x_{q}\right):=\left[\prod_{j=1}^{q-1}\left(1+x_{j}+x_{j+1}\right)\right]\left(a+x_{1} x_{q}+x_{1}+\cdots+x_{q}\right) / x_{1} x_{2} \ldots x_{q}$.

For $q \geq 6$, the difficulty is to prove the (UCP) property, the problem remains open.

Acknowledgment. Thanks to Jacques Lafontaine (Montpellier, France) for fruitful dicussions about Fact 4.

\section{REFERENCES}

[1] E. Barbeau, B. Gelbord and S. Tanny, Periodicities of solutions of the generalized Lyness recursion, J. Difference Equ. Appl., 1 (1995) 291-306.

[2] G. Bastien and M. Rogalski, Global behaviour of the solutions of Lyness' difference equations, J. Difference Equ. Appl., 10 (2004), 977-1003. 
[3] G. Bastien and M. Rogalski, On some algebraic difference equations $u_{n+2} u_{n}=$ $\psi\left(u_{n+1}\right)$ in $\mathbb{R}_{*}^{+}$, related to families of conics or cubics: generalization of the Lyness' sequences, J. Math. Anal. Appl., 300 (2004), 303-333.

[4] G. Bastien and M. Rogalski, On the algebraic difference equation $u_{n+2} u_{n}=\psi\left(u_{n+1}\right)$ in $\mathbb{R}_{*}^{+}$related to a family of elliptic quartics in the plane, Adv. Difference Equ., 3 (2005), 227-261.

[5] G. Bastien and M. Rogalski, On the algebraic difference equations $u_{n+2}+u_{n}=$ $\psi\left(u_{n+1}\right)$ in $\mathbb{R}$, related to a family of elliptic quartics in the plane, J. Math. Anal. Appl., 326 (2007), 822-844.

[6] G. Bastien and M. Rogalski, Results and conjectures about order q Lyness' difference equation $u_{n+q} u_{n}=a+u_{n+q-1}+\ldots+u_{n+1}$ in $\mathbb{R}_{*}^{+}$, with a particular study of the case $q=3$, Adv. Difference Equ., 2009, article ID 134749.

[7] G. Bastien and M. Rogalski, On the topological nature of the stable sets associated to the second invariant of the order $q$ standard Lyness' equation, Sarajevo J. Math., 7 (19) (2011), 1-8.

[8] S. Cairns, An elementary proof of the Jordan-Schönflies Theorem, Proc. Amer. Math. Soc., 2 (6) (1951), 860-867.

[9] A. Cima, A. Gasull and V. Manosa, On two and three periodic Lyness difference equations, J. Difference Equ. Appl., 18 (5) (2012), 849-864.

[10] A. Cima, A. Gasull and V. Manosa, Dynamics of the third order Lyness' difference equation, J. Difference Equ. Appl., 13 (10) (2007), 855-884.

[11] J. Esch and T. D. Rogers, The screensaver map: Dynamics on elliptic curves arising from polygonal folding, Discrete Comput. Geom., 25 (2001), 477-502.

[12] A. Gasull, V. Manosa and X. Xarles, Rational periodic sequences for the Lyness recurrence, Discrete Cont. Dyn. Syst. Ser. A, 32 (2) (2012), 587-604.

[13] E. J. Janowski, M. R. S. Kulenović and Z. Nurkanović, Stability of the $k$-th order Lyness' equation with a period-k coefficient, Int. J. Bifurcations Chaos, 17 (1) (2007), $143-152$.

[14] M. R. S. Kulenović, Invariants and related Liapunov functions for difference equations, Appl. Math. Lett., 13 (2000), 1-8.

[15] J. Lafontaine, Introduction aux variétés différentiables, Presses Universitaires de Grenoble, Grenoble (1996).

[16] W. Rudin, Real and Complex Analysis, second edition, McGraw-Hill, 1974.

[17] E. C. Zeeman, Geometric unfolding of a difference equation, unpublished paper: preprint, Hertford College, Oxford, 1996.

(Received: June 7, 2012)

(Revised: October 22, 2012)
Gay Bastien

Institut Mathématique de Jussieu

(CNRS and Université Pierre et Marie Curie)

Marc Rogalski

Laboratoire Paul Painlevé

(CNRS and Université des Sciences et

Technologies de Lille) and

Institut Mathématique de Jussieu

Université Pierre et Marie Curie

F-75252 Paris Cedex 05, France

E-mail: marc.rogalski@upmc.fr 